\title{
Prosumo, swarming y transmedia. Hacia un nuevo concepto de stakeholder
}

\section{Prosum, swarming and transmedia. Towards a new concept of stakeholder}

\author{
Eugenio Moya \\ Catedrático de Filosofía \\ (Universidad de Murcia)
}

Julia Moya

Investigadora doctoral en el Programa de doctorado en gestión de la información y de la comunicación en las organizaciones.

(Universidad de Murcia)

Fecha de recepción: 22 de mayo de 2018

Fecha de revisión: 22 de junio de 2018

Fecha de publicación: 1 de julio de 2018

Para citar este artículo: Moya, E. y Moya, J. (2018). Prosumo, swarming y transmedia.

Hacia un nuevo concepto de stakeholder, Icono 14, 16 (2), 25-50. doi: 10.7195/ri14.

v16i2.1213 


\section{Resumen}

La narrativa transmedia representa hoy una auténtica revolución comunicativa. También un nuevo modelo de negocio. Un nuevo paradigma en el modo de generar y hacer circular los contenidos; la mejor expresión de las redes tecnocomunicativas digitales, unas redes que, al propiciar entornos colaborativos, donde se hace realidad el doing it together, han creado vínculos más estrechos entre los productores de bienes y servicios, las agencias publicitarias, los medios y el público. Internet y las redes sociales han empoderado a los ciudadanos comunes (prosumidores y pronet@rios) y han obligado a las organizaciones $\mathrm{y} / \mathrm{o}$ marcas a conocer sus intereses y valores a fin de crear conversaciones con ellos, auténticos stakeholders. Ellos no aportan solo opinión; son auténticos inversores. Sus aportaciones, muchas veces intangibles, llegan a ser tan importantes para las marcas/empresas como las económicas que realizan los accionistas. Hablamos de inversiones emocionales, sociales e intelectuales.

En este artículo, presentamos un marco conceptual que permite generar una magnitud intensiva, un grado, en función del continuo participativo que va desde la escasa a la alta inversión. Consideramos que la clave para medir el éxito comunicativo o la eficacia publicitaria radica en esclarecer las gratificaciones que obtienen los prosumers al participar del mundo narrativo y de la estrategia comunicativa de las marcas. Son aquellas las que les hacen comprometerse, hasta su identificación, en la transmedial world-building de las organizaciones.

Palabras clave: Internet - Multitud inteligente - Prosumidor - Quinto poder Sistema autopoyético - Transmedia

\section{Abstract}

Transmedia storytelling represents today a genuine communicative revolution. Also a new business model. A new paradigm in the way to generate the contents; the best expression of digital technocommunication networks, networks that foster collaborative environments, where doing it together become reality, have created closer links between producers of goods and services, advertising agencies, the media and the public. Internet and social networks have empowered common citizens (prosumers and pronet@rians) and have forced organizations/brands to make their interests and values in order to create conversations with them, authentic stakeholders. They don't provide only opinion; are 
genuine investors. Their contributions, often intangible, become so important for brands/ companies as the economic investment made by stockholders. We are talking about emotional, social and intellectual investments.

In this paper, we present a conceptual framework that allows generating an intensive magnitude, a degree, depending on the continuous participatory process that goes from the scarce to the high "investment". We consider that the key to measure the success of communication or advertising effectiveness lies in clarifying the gratifications that get the prosumers to participate in the world narrative and the communication strategy of the brands. Are those that make them commit, until their identification, in the transmedial world-building of organizations.

Key Words: Autopoietic system - Fifth estate - Internet - Prosumer-Smart swarm - Transmedia

\section{Introducción: más allá del consumo, la emergencia del quinto poder}

Estos dos tuits, publicados el 17 de febrero de 2017, tuvieron un eco extraordinario en la prensa.

\section{Hero Baby \\ @HeroBaby}

Seguir

A @samantavillar, acomodada y famosa, sus hijos le hacen "perder calidad de vida". Ánimo Samanta, tus hijos te querrán igual. \#EmpiezaLaVida

$15: 40$ - 17 feb 2017

$306 \quad 395$

Imagen 1

samantavillar

@samantavillar

Seguir

Hola @HeroBaby Por que metéis aceite de palma en vuestros productos, con la baja calidad nutricional y el perjuicio al ambiente? Gracias

21:03 - 17 feb 2017

$1.360 \quad 1.805$

Imagen 2 
El diario Público informaba, en efecto, el 20 de febrero que «La marca de confituras Hero se 'pega un tiro en el pie' al atacar a la periodista Samanta Villar». Noticias parecidas pudieron ser leídas u oídas ese mismo día en otros diarios y medios de comunicación: La Vanguardia, Europapress, Cadena Ser... Todos ellos informaban de que la empresa murciana Hero Baby, el mismo día en que lanzaba la campaña \#EmpiezaLaVida, en la que hablaba de la aventura de tener hijos («Cuando tienes un hijo no acaba lo bueno, empieza la aventura más grande»), había cometido el error de publicar un tuit en el que, criticando abiertamente el libro de Samanta Villar Madre hay más que una (2017), señalaba: «A @samantavillar, acomodada y famosa, sus hijos le hacen 'perder calidad de vida'. Ánimo Samanta, tus hijos te querrán igual». El error estaba en que los internautas interpretaron que se trataba de una pugna desigual entre una multinacional y una escritora (una versión 2.0 de la pugna entre Goliat y David) y se posicionaron, abierta y mayoritariamente, a favor de la periodista. Máxime cuando la denuncia que ella hacía no fue desmentida por la marca ${ }^{1}$.

El caso revela dos fenómenos comunicativos actuales; primero: los mass media tradicionales (prensa, radio, televisión...) sobreviven adecuando sus formatos y gran parte de sus contenidos a medios digitales como Twitter o Facebook; segundo: en los nuevos medios ha emergido un nuevo tipo de consumidor que, lejos de su pasividad tradicional, ha pasado a ser actor imprescindible en las estrategias organizativas y comunicativas de empresas e instituciones. De hecho, las instituciones y organizaciones económicas, sociales, políticas y culturales tradicionales exitosas saben que en la era digital los ciudadanos comunes tienen un nuevo papel en sus estrategias comunicativas y publicitarias. Hero Baby olvidó la lección fundamental: el consumidor «puede representar una fuerza poderosa cuando, devolviendo el golpe económicamente a las principales instituciones, puede condicionar directamente su poder e influencia» (Jenkins, 2008, p. 224).

Hasta la Era de Internet, los trabajos teóricos y creativos de la comunicación y la publicidad (entre otros, los de Bernays, Lasswell y McLuhan) alimentaron la idea de los efectos todopoderosos de los mass media en la opinión pública y el consumo; una idea que, aunque fue sometida parcialmente a revisión por los análisis de Paul F. Laserfeld y su equipo de la Universidad de Columbia en los años cuarenta 
del siglo pasado, siguieron siendo decisivas para determinar lo que los ciudadanos o consumidores debían hacer con su voto o con su dinero (Bernays, 2008, p. 15). De hecho, las estrategias de imagen de empresas, partidos y gobiernos evolucionaron adaptándose a los requerimientos de los medios «fordistas» de comunicación. Del conocido como cuarto poder. Pero, como ha señalado Dutton, estamos asistiendo a la emergencia del fifth estate:

The rise of the press, radio, television and other mass media enabled the development of an independent institution: the 'Fourth Estate', central to pluralist democratic processes. The growing use of the Internet and related digital technologies is creating a space for networking individuals in ways that enable a new source of accountability in government, politics and other sectors. This paper explains how this emerging 'Fifth Estate' is being established and why this could challenge the influence of other more established bases of institutional authority. (Dutton, 2009, p. 1).

Pues bien, este trabajo, pretende elevar a categoría el caso Hero Baby para mostrar, en primer lugar, que es la arquitectura de las redes digitales la que en gran medida ha permitido el empoderamiento del ciudadano común (pronet@ rio $^{2}$ y prosumidor ${ }^{3}$ ). Como Dutton, Jenkins (Jenkins et al., 2016) o Castells (2015), argumentaremos que la nueva base tecnológica ha permitido a ese ciudadano la capacidad de acceder sin demasiadas restricciones y sin intermediación a la información, su manejo y circulación, con lo que ha podido hacerse oír al margen de las rutas obligadas que marcaron en el fourth estate los poderes políticos, económicos y culturales. Y la consecuencia es obvia: el éxito de las organizaciones depende de su capacidad de tomar sus modelos organizativos de las comunidades populares de fans (Jenkins, 2008, p. 33). En este sentido, nuestro segundo gran objetivo, es presentar un marco conceptual que permite generar una magnitud intensiva, un grado, que ayude a medir en un continuo participativo el grado en que el prosumidor se compromete en el mundo narrativo y la estrategia comunicativa de las marcas para terminar convirtiéndose en un nuevo stakeholder. 


\section{Metodología: un enfoque sistémico y cognitivo de la comunicación}

Toda red está compuesta de nodos interconectados. Estos pueden ser más o menos centrales en la red, pero los nodos no son centros sino enlaces; solo existen y pueden funcionar como componentes de la red. La red es la unidad. Por otro lado, es la conectividad (transitividad) de los nodos la que determina su posición en la red, o sea, su influencia, relevancia o prominencia. Christakis y Fowler han mostrado que la centralidad es un valor asignado al nodo debido a su posición estructural en la red y su capacidad de "contagio social" (Christakis y Fowler, 2010, pp. 1-2). Podemos distinguir, así, entre redes centralizadas y descentralizadas. En las primeras, todos los nodos, menos uno, son periféricos y sólo pueden comunicarse a través del nodo central. La caída del mismo priva del flujo informativo a todos los demás nodos. Son, por tanto, jerárquicas, piramidales.

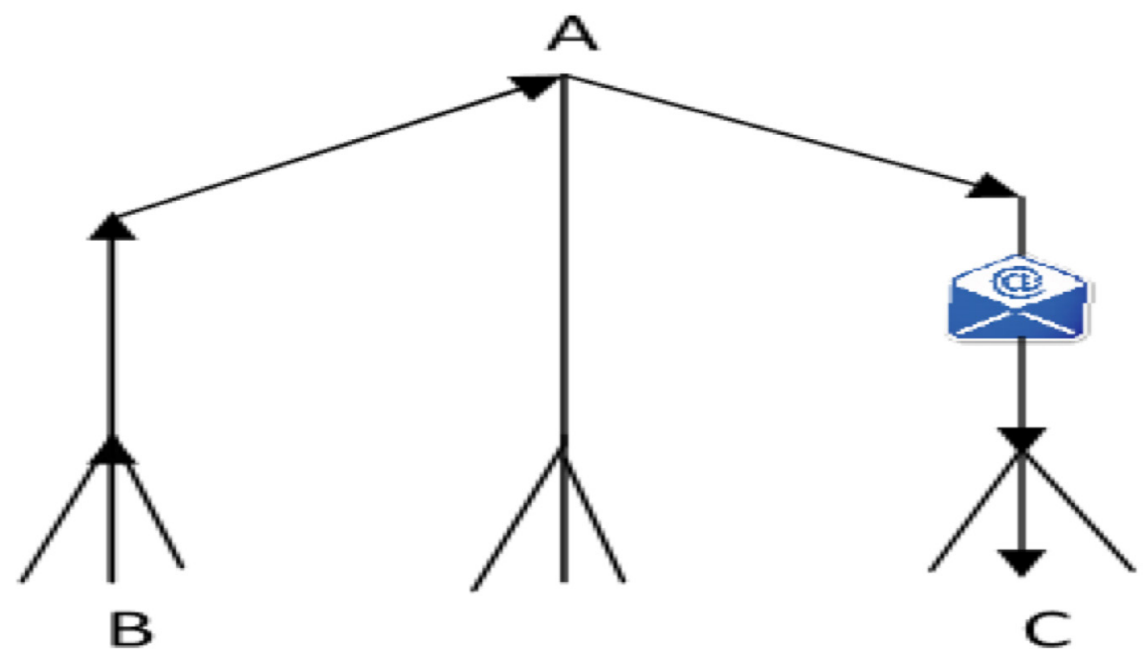

Figura 1: Red centralizada.

La traducción mediática de estas redes centralizadas supone que todos los nodos, menos uno, son periféricos. La caída de aquel priva de flujo informativo a todos los demás nodos. Es el modelo mantenido desde los años 20 por los teóricos de comunicación, ya sea en la variante «hipodérmica» desarrollada por Bernays, Lasswell y luego rectificada en los cincuenta por Katz y Lazarsfeld (1955), que 
acentuaba el papel de los líderes de opinión- ya en la ecológica -desarrollada desde los setenta hasta hoy por McLuhan, Ong, Derrick de Kerkhove, etc.- que piensa los medios de comunicación como ambientes selectivos que dan forma al receptor. En ambos casos, podemos hablar de un mismo paradigma; defienden la idea de la fabricación mediática de la realidad. El siguiente diagrama muestra bien lo esencial de este enfoque:

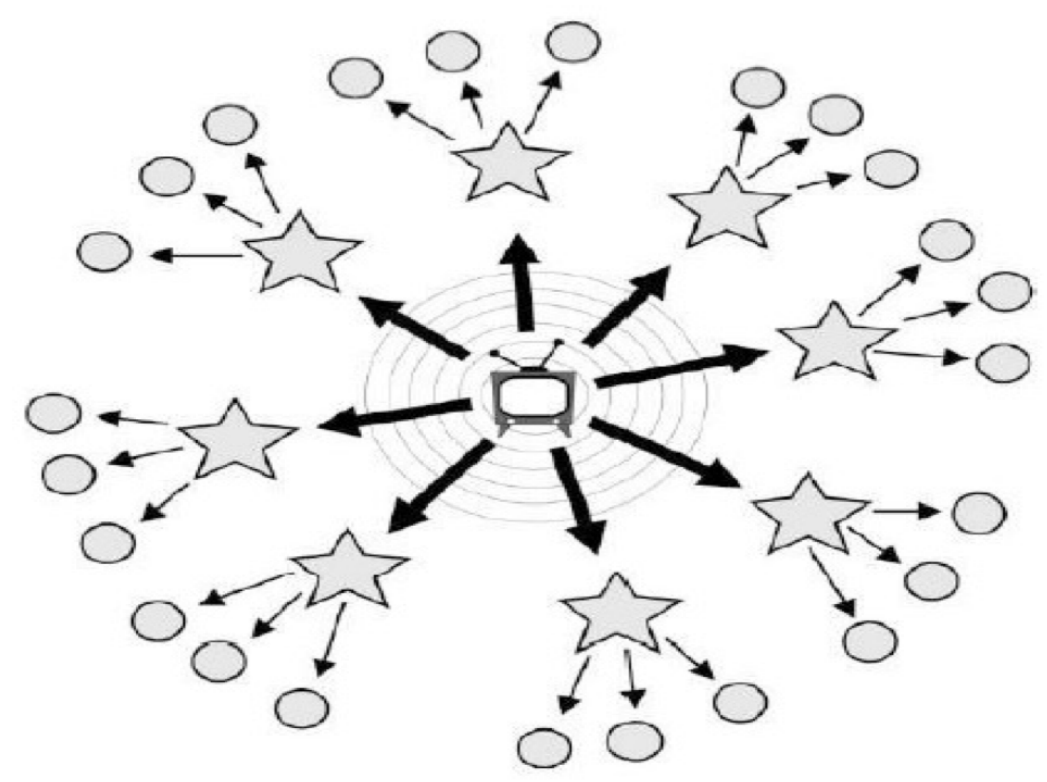

Figura 2: Las estrellas representan los líderes de opinión y los círculos los receptores (Watts y Dodds, 2007)

El enfoque causal y selectivo de la comunicación no es, sin embargo, adecuado para dar cuenta de las nuevas redes del informacionalismo. Hace suya la idea de un emisor activo y un receptor pasivo que, compartiendo un código y contexto, limitan su interacción comunicativa (mensaje) a la lógica acción-reacción impuesta por el medio y la fuente. Pero, Internet o las redes sociales son redes descentralizadas; no existe en ellas un único nodo central, a pesar de que pueden existir nodos hiperenlazados (hubs), con lo que la caída de uno no tiene por qué llevar a una ruptura o desaparición de la red. Hablamos de redes multicentradas, en las que ningún nodo tiene poder de filtro informativo central. Desaparece, por tanto, la divisoria entre centro y periferia, propia de las redes «fordistas». 


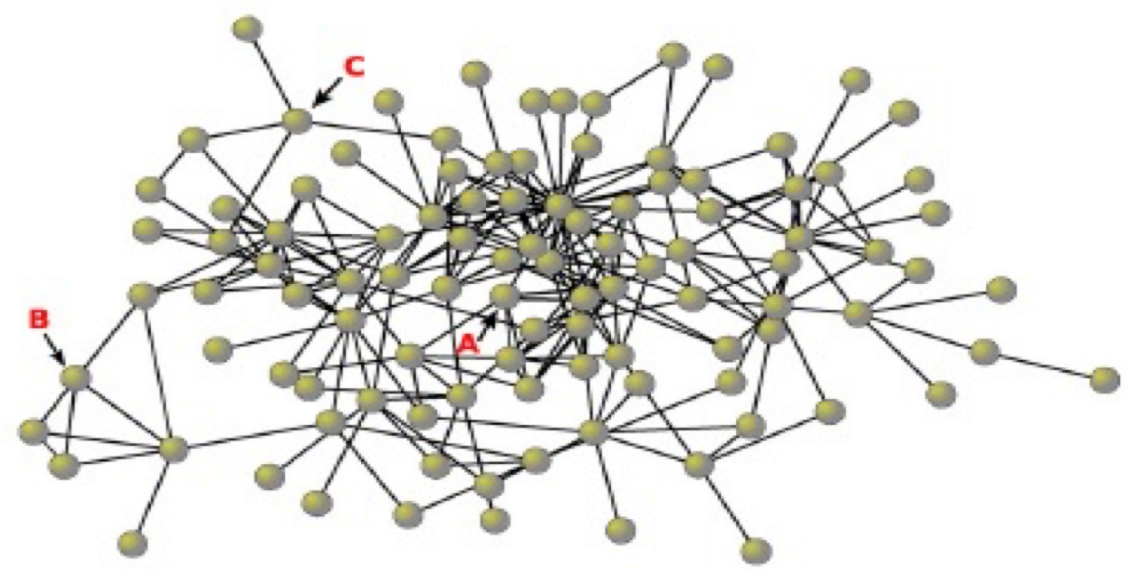

Figura 3: Red descentralizada. (Christakis y Fowler, 2010, p. 2).

Las nuevas redes están pensadas para la comunicación multidireccional, no son medios generalistas de comunicación de masas. Es cierto que las redes económicas, políticas y culturales del industrialismo comunicaron centro y periferia, pero su capacidad para introducir nuevos actores y contenidos en el proceso de organización social, al margen de los centros de poder, fue siempre muy limitada. Siempre requirieron la intermediación, con lo que las redes centralizadas evolucionaron hacia organizaciones poco participativas. Cosa bien distinta ocurre en las redes digitales. Ellas han tensionado la lógica de las organizaciones de producción, poder y cultura; han desencadenado fuerzas creativas al margen de los productores, gerencias de los medios, consejos editoriales o élites culturales. No es extraño que empiece a hablarse medios de multitudes inteligentes.

Joe Trippi, que fue el director de la innovadora campaña electoral de Howard Dean en las presidenciales estadounidense de 2004, habló de un creciente empoderamiento de ciudadanos comunes que disputan a los poderes políticos y económicos el nuevo capital: la información.

EI poder se está desplazando desde las instituciones que siempre han dirigido de arriba-abajo, acumulando piramidalmente la información y diciéndonos cómo dirigir nuestras vidas, hacia un nuevo paradigma de poder democráticamente distribuido y compartido por todos nosotros. (Trippi, 2004, p. 4). 
Necesitamos, pues, nuevos enfoques metodológicos para dar cuenta del cambio de paradigma. Consideramos que el papel del prosumidor exige un enfoque sistémico, dinámico y holístico de la comunicación; entendiéndola como un todo integrado por subsistemas que presentan autonomía -ya que tienen una forma determinada y actúan conforme a unas reglas propias-, pero que coevolucionan interactuando (Maturana y Varela, 2004, pp. 53-54). Entendemos, en consecuencia, la marca como un activo intangible que evoluciona positiva o negativamente en el tiempo en función de las dinámicas conversacionales expansivas o depresivas que se generen entre los diversos actores: medios, organizaciones, narrativas y prosumidores (Moya, 2017, p.21).

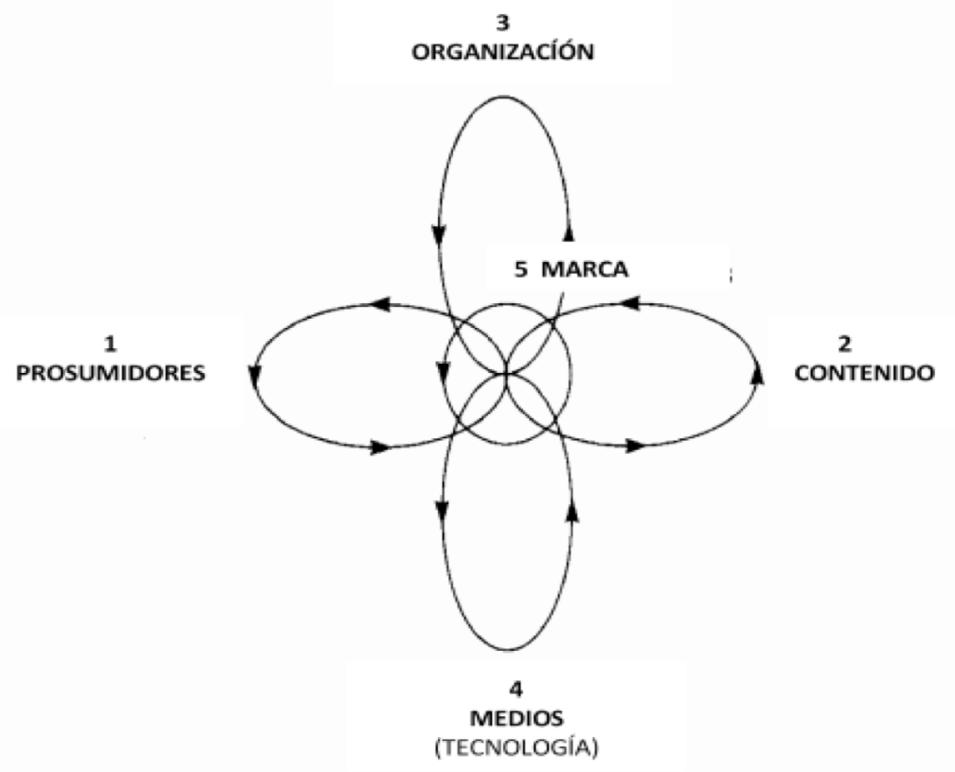

Figura 4: Modelo sistémico, dinámico y conversacional de las marcas.

No hablamos de sistemas adaptativos complejos, sino de sistemas autopoyéticos dinámicos, o sea, autoorganizados ${ }^{4}$. En definitiva, la comunicación debemos entenderla como una red que evoluciona expandiéndose o reduciéndose por la interacción en el tiempo de diferentes «actores» que, como (sub-)sistemas abiertos a la información, establecen relaciones de cooperación y/o competencia. 


\section{La inteligencia de los enjambres. El fenómeno swarming}

Hemos hablado de empoderamiento, pero, en una época en la que corporaciones de medios globales como Google y Facebook extraen miles de millones de dólares de plusvalor explotando la información autogenerada, cultural y biométrica por sus usuarios, ¿podemos hablar de una auténtica prosumer revolution?

Desde el punto de vista económico, el prosumo comienza, en cualquier caso, a dar nombre, incluso, a un nuevo tipo de microeconomía, para algunos revolucionaria, con efectos macro: la Prosumer Economy, también Wikinomics (Tapscott y Williams, 2009), un modo de producción colaborativa, que socializa el conocimiento en redes paritarias, P2P, e impresión 3D. Sin embargo, no faltan perspectivas más críticas. Es el caso de Byung-Chul Han. Para él, el «enjambre digital» hay que pensarlo como una nueva tecnología social: la psicopolítica, que, a través de los big data, permite a poderes económicos y políticos colonizar las mentes de los individuos, avanzando desde una vigilancia pasiva hacia un control activo (Han, 2014, p.25). Para Han, cuando un mensaje político o publicitario nos hace llegar un producto por el que nos hemos interesado, en realidad el producto somos nosotros. El prosumer se autoconcibe como empresario de sí mismo, pero las retóricas emancipatorias, revolucionarias, no son para él simples ilusiones neoliberales. Él, vendría a concluir Han, no es verdadero sujeto, agente, sino una víctima del imperativo publicitario neoliberal de optimización personal que solo tiene un telos: reducción de costes $\mathrm{y}$, por tanto, mayor rentabilidad.

Negarle toda verdad a la hipótesis de Han sería un error. La prosumer economy toma forma en la intersección de tres tendencias: la tecnológica, que permite a cualquier interlocutor, sin mucho esfuerzo y casi sin equipamiento, obtener, almacenar y hacer circular la información de un modo y a una velocidad impensable; la mediática: las nuevas redes tecnocomunicativas han posibilitado un networked individualism que compite con empresas y agencias publicitarias (o con grupos de comunicación y políticos), en la producción colectiva de lo que hoy constituye el mayor activo económico, político y cultural: el contenido informativo (imágenes, textos, música, relatos...); y la económica: el capitalismo, en su tendencia a aumentar beneficios, externaliza costes y, para ello, compromete a los consumidores, con la ayuda impres- 
cindible de las nuevas tecnologías y medios digitales. Sin embargo, Han soslaya el carácter ambivalente de los nuevos fenómenos.

En efecto, Jenkins y otros (Jenkins, Ito y Boyd, 2016; Herrero, et al., 2016) han señalado la emergencia de un novísimo conjunto de prácticas individuales y comunales de producción e intercambio económico, político y cultural. Son prácticas usadas en las redes para muchos fines y por muchos agentes, pero no puede desconocerse que encarnan un conjunto de ideales sobre cómo facilitar el aprendizaje, el empoderamiento y la acción cívica.

Coincidimos con ellos. La cultura participativa y su nuevo ethos: doing it together, más que doing it yourself, revela un fenómeno social ligado a los comportamientos (económicos, epistémicos y políticos) colectivos en entomos colaborativos: the swarming. Es una smart swarm bien estudiada en sistemas biológicos de diferente índole: bancos de peces, termiteros, bandadas de aves, colonias bacterianas, etcétera. Estos enjambres muestran una ventaja que no ha sido atendida por Han: en los enjambres se optimizan las estimaciones y comportamientos individuales. En el caso de las termitas, nos encontramos con una destacable habilidad del grupo a la hora de encontrar el camino más corto entre el alimento y el termitero. La razón es sencilla: al moverse individualmente van dejando rastros de feromona detectables por otras termitas, con lo que colectivamente terminan eligiendo como mejores caminos aquellos marcados por la más alta concentración de feromonas. Un fenómeno análogo ocurre con la migración de aves o con el comportamiento de los bancos de peces:

However, in principle, collective intelligence may also emerge from interactions between individuals, rather than from the enhancement of personal estimates. Here, we reveal that this emergent problem solving is the predominant mechanism by which a mobile animal group responds to complex environmental gradients. Robust collective sensing arises at the group level from individuals modulating their speed in response to local, scalar, measurements of light and through social interaction with others. This distributed sensing requires only rudimentary cognition. (Berdahl. et al., 2013, p. 574). 
¿Podríamos decir que son distintos nuestros comportamientos colectivos tras las evaluaciones individuales que hacemos de hoteles o restaurantes en TripAdvisor 0 nuestras colaboraciones en la Wikipedia? $¿ 0$ que es distinto nuestro comportamiento colectivo cuando cliqueamos like en Facebook o nos hacemos followers en cualquier web o red social? Por supuesto que no. Los medios digitales de comunicación, por su misma lógica o arquitectura, inducen tres fenómenos básicos de inteligencia colectiva: socializan el conocimiento; permiten la coordinación de los miembros de grupos establecidos optimizando sus estimaciones; $y$, por último, fomentan la cooperación o integración de individuos en colectivos inteligentes.

No es extraño, por ello, que desde diferentes áreas -incluidas las matemáticasse aborde el problema de la credibilidad de la información analizando aplicaciones de la tecnología de teléfonos inteligentes y de cómo diferentes usuarios informan sobre eventos del mundo físico: búsqueda y rescate, lucha contra incendios... (Liu et al., 2012; Jiang et al. 2017).

A diferencia de los espacios de inteligencia monológica (libro, prensa escrita, radio, televisión), el social swarming permite obrar colectiva y descentralizadamente a fin de optimizar respuestas y resolver problemas complejos, aunque los agentes individualmente no acrediten capacidades extraordinarias.

\section{Marca y narrativa transmedia}

La crisis de la idea de mediación y la emergencia de los prosumidores ha generado nuevas vías de participación, además de novedosas posibilidades narrativas. Entre ellas, qué duda cabe, la storytelling transmedia. Ella representa un nuevo paradigma en el modo de generar y hacer circular los contenidos. Y es precisamente su auge lo que refuerza la idea de que el alcance y éxito de una estrategia de comunicación corporativa, coordinada y unificada depende del mix: contenido + inteligencia colectiva + participación de la audiencia.

El concepto transmedia fue acuñado por Jenkins en 2003. Lo hizo en un artículo publicado en la revista Technology Review. En él, Jenkins afirma que participamos en una nueva era de convergencia (de plataformas, de medios y de lenguajes), donde inevitablemente los contenidos se distribuyen a través de múltiples canales. Para 
ejemplificarlo, analiza numerosos ejemplos emblemáticos de producciones transmediáticas como Star Wars, Matrix y Harry Poter. En todo caso, antes de que Jenkins lo popularizara, el término transmedia fue usado por primera vez en 1975 por Stuart Saunders Smith. Él creó una composición musical que bautizó de transmedia music. Mezcló en una única composición musical distintas piezas musicales producidas por diferentes instrumentos en una variedad de estilos. Cada música por separado tenía su unidad, pero, en conjunto, constituía otra composición. Poco más de una década después, la investigadora Marsha Kinder (1991) creó algo a partir del concepto de Smith y la idea de polifonía, propuesta por los lingüistas, la denominada intertextualidad transmedia, para referirse sobre todo a las relaciones que se producían entre televisión, cine, videojuegos y juguetes, «as compatible members of the same everexpanding supersystem of mass entertainment» (Kinder, 1991, p. 40). Intertextualidad era algo así como intermedialidad. Pero no se trataba de verter un contenido en diferentes medios para reforzarse entre sí. Una red intertextual, siendo la condición necesaria, no es suficiente para hablar de narración transmediática. Frente a la idea de One Story, Many Media (Dowd, 2015) la gran aportación de Jenkins fue, justamente, señalar que cada medio debía hacer un aporte exclusivo, distintivo y valioso a la historia; es decir, no se trata de una adaptación o traducción intersemiótica, sino de que en cada medio se ofrece un contenido nuevo narrativamente hablando.

En este sentido, el papel de los prosumidores resulta estratégicamente crucial. Ellos re-crean contenidos; los modifican; los expanden; trabajos reservados hasta hace poco a las gerencias de los medios o las agencias publicitarias. En palabras de Jenkins (2010, p. 948), «shaped top down by decisions made in corporate boardrooms and bottom up by decisions made in teenagers bedrooms». De hecho, en una narrativa transmedia.

1. Hay una narrativa-canon creada por uno o varios visionarios contratados para la marca y que no tienen por qué ser profesionales.

2. El relato se expande por una variedad de medios y plataformas. Al menos tres.

3. Se analiza la fortaleza de cada medio para determinar dónde se cuentan las distintas partes del relato.

4. El usuario (prosumidor) termina siendo coparticipe en la expansión de las narrativas originarias (relatos-canon) promovidos por las organizaciones. 
5. Se procure siempre la coherencia de los contenidos e historias co-creados, evitando que las historias introduzcan fracturas, incoherencias.

Recordemos que uno de los primeros ejemplos de narrativa transmedia en el mundo fue Matrix, que tuvo su primera entrega de 1999 (Jenkins, 2008, p. 99). La historia no se limitó a las tres películas que fueron distribuidas en los cines, sino también a dos videojuegos, un portal específico en Internet y nueve cortometrajes animados. Por si fuera poco, mostraba los riesgos existenciales que vivían los propios cibernautas en sus primeros pasos digitales. En definitiva, un universo formado por pequeñas historias que fueron presentados utilizando diversos formatos y tecnologías y conectando con las experiencias de la audiencia. Así, los esfuerzos corporativos y las respuestas de base terminaron reforzándose en las narrativas transmediáticas, creándose así vínculos más estrechos entre los productores, los contenidos y los prosumidores.

Que las organizaciones sean capaces de generar dinámicas expansivas depende, en último término, de su capacidad para establecer conexiones estratégicas, a medio-largo plazo, con el prosumidor, a fin de crear una conversación y una relación leal a medio-largo plazo con él que permita acercarse a su corazón, conocer sus motivaciones, valores y sentido de la vida; en definitiva, para, finalmente, convertirlo en fan.

Una simple búsqueda en Google de los clubs de fans de La Guerra de las Galaxias revela que la Vader's Fist o Legión 501 es una organización internacional de trajes de Star Wars, integrada y dirigida por fans de la saga, que, aunque no está patrocinada por Lucasfilm Ltd., promociona el vestuario imperial preferido de Lucasfilm. Star Wars, sus personajes, vestuario y todo lo relacionado son, de hecho, propiedad intelectual de Lucasfilm Ltd. Evidentemente, son los trajes usados en la Academia Jedi Mundial: una verdadera Orden Jedi, creada para todas las edades y lugares del planeta, una orden que vive, piensa y actúa como los personajes de ficción. Y aquí está la clave: la mezcla de ficción y realidad es tal que las fronteras se difuminan. Es lo que los estudiosos de la comunicación han llamado, con Jenkins, la extractabilidad (extractability): la capacidad que tiene la narrativa transmedia para favorecer, gracias a mecanismos de inmersión, la toma de cosas de la ficción para 
aplicarlas a la vida real (Krzysztof, 2015, pp. 83-96). Los fans son «evangelizadores a tiempo completo», no pierden la ocasión de promover su narrativa favorita; se convierten, así, para las organizaciones en auténticos stakeholder de pleno derecho.

\section{No targets. Los prosumidores como stakeholders}

Fue Milton Friedman, en Capitalism and freedom (1962), quien estableció la teoría de que, estrictamente, las empresas solo están en deuda con sus accionistas (stockholders). Para él, los agentes económicos persiguen siempre y solamente un objetivo: maximizar su utilidad. (Friedman, 1962, p. 133).

Desde los finales de los setenta, E. Freeman introdujo el concepto de stakeholder para reformular la noción de utilidad. Comenzó a hablar de la responsabilidad social corporativa, pero la ampliación trajo consigo complejidad e indefinición conceptual, pues ¿a quién cabría considerar stakeholder? El propio Freeman ha variado su respuesta desde sus primeras publicaciones ${ }^{5}$. Sin embargo, es identificable su respuesta principal: stakeholders son aquellos grupos que pueden afectar o ser afectados por el logro de los propósitos de la organización. Evidentemente, podemos diferenciar entre stakeholders, según sea su influencia directa o indirecta sobre la empresa: los primarios y los instrumentales. Los primarios son esenciales para la supervivencia de cualquier empresa, mientras los stakeholders instrumentales están en el entorno amplio de la empresa y son aquellos que pueden influir en los primarios. Entre los primarios estarían los inversores, los productores, reguladores y consumidores; entre los segundos, los competidores, los medios de comunicación o los activistas.

En principio, esta tipificación no ofrece problemas desde el punto de vista del marketing. Sin embargo, desde el punto de vista comunicativo y publicitario tiene debilidades considerables. La principal radica en el lugar que ocupa el público (target), aquel que es potencial comprador, pero que se limita finalmente a merodear en una web corporativa; a visionar un tráiler, pero no consume la película, o que comparte un contenido en una red social. Pensemos que, como Scolari (2010, p. 84) ha señalado, las expansiones narrativas del texto base en la narrativa transmedia pueden ser implementadas con microrrelatos vinculados a formatos promocionales: 
tráileres, recapitulaciones, vídeos sincronizados, etc., formatos que en ocasiones son inventados y popularizados por el fandom. Pero, incluso, nos encontramos con formas transversales, integradas por textos terciarios: los producidos en las discusiones y los comentarios de la audiencia sobre los productos cinematográficos, televisivos u otros.

Marcelo Baro (2011, p. 140), ha intentado, por ello, precisar más. Considera que son tres los atributos de los stakeholders: poder, legitimidad y urgencia. Tienen poder cuando de hecho pueden influir en las decisiones organizacionales, cuando pueden forzar a una organización a hacer algo que de otra manera no hubiera hecho. Tienen legitimidad cuando en derecho les corresponde exigir un determinado comportamiento a la organización. Finalmente, tienen urgencia cuando sus demandas deben ser atendidas. En consecuencia, no debe entenderse por stakeholder cualquier grupo que afecta o pueda ser afectado por la actividad de la organización.

Sin embargo, la viralidad, la vecindad y el contagio digital -o sea las dinámicas propias de las nuevas redes tecnocomunicativas- nos obligan a hacer del concepto de stakeholder un concepto lógicamente borroso. No es posible hoy determinar la pertenencia o no pertenencia por los vínculos operativos con la organización, que puede materializarse en un título, un contrato, una orden de compra o un convenio. La revolución digital ha creado un nicho tecnoeconómico que obliga a las organizaciones no solo a situar como departamento estratégico a los gabinetes de comunicación. Esto ya ocurría en el industrialismo y la sociedad de los mass media. Las ha obligado a entablar conversaciones con el público para analizar sus percepciones y experiencias con las marcas. El público forma parte ya de la «cultura organizativa» (Fernández y Bajo, 2012). Tiene poder, legitimidad y urgencia.

Wilson Rickerson y su equipo de investigación, en un report reflexivo y pormenorizado (por países) para la International Energy Agency (IEA), ha analizado incluso los efectos políticos de un público, que lejos de ser un simple cliente potencial, prosume en el sector eléctrico, y su conclusión es clara:

As with the introduction of any new business model, the emergence of prosumers creates winners and losers, depending on how the incentives of different 
stakeholders are aligned. The alignment (or conflict) of stakeholder interests can most readily be influenced through policy and regulation as will be discussed throughout this report. However, policy makers will likely face conflicting political pressures from different stakeholders as they attempt to determine the most appropriate prosumer strategy. (Rickerson, et al., 2014, p. 36).

Los prosumidores deben ser considerados así como fuzzy stakeholders (Kumar y Kumar, 2015, p. 205). Realizan inversiones en las marcas/empresas que muchas veces son cuantitativamente intangibles, pero que son, desde el punto de vista económico, tan importantes o más como las de los accionistas. Hablamos de inversiones emocionales, sociales o intelectuales. Por eso, conocer sus motivaciones y opiniones no solo cuenta. Son hoy decisivas para el éxito de las organizaciones.

El objetivo es claro: lograr el máximo engagement del público, un público que buscará gratificaciones asociadas a la satisfacción de sus necesidades múltiples. Evidentemente, ya no basta con analizar targets para segmentarlos en función de su edad, género, nivel económico. Las infotecnologías transversalizan diversos segmentos y nos obligan a una tarea de perfilación que es cuantitativa, pero que no puede dejar de ser cualitativa. Esta es nuestra propuesta. Necesitamos enfoques cognitivos, hermenéuticos y no solo analíticos para dar cuenta de un concepto viable de eficacia comunicativa y publicitaria.

Nosotros presentamos aquí un marco conceptual que permite generar una magnitud intensiva, un grado, en función de un continuo participativo, que va desde la escasa a la alta participación ${ }^{6}$. En este marco, la importancia radica en esclarecer qué es lo que lleva a los prosumers a formar parte del mundo narrativo, de la estrategia comunicativa, de las marcas (organizaciones). Es un enfoque sistémico y cognitivo, heredero de las teorías de la comunicación, la TUG, elaborada por Blumler, Katz y Gurevitz (1973), que no se interesaron por el efecto que tienen los medios de comunicación en las creencias, valores o conductas de las personas, sino por el efecto que tienen las personas en los medios de comunicación y los mensajes. Asumieron de forma pionera que las audiencias, incluso en los medios masificadores, no son reactivas, como pensaron los conductistas, o sea, consumidores pasivos de medios y productos. Los públicos tenían, al menos, un poder selectivo sobre su consumo de contenidos y 
plataforma. Sus motivaciones, sus valores; en definitiva su "psicología", resultaba, y resulta, de vital importancia para generar dinámicas comunicativas expansivas.

Según la TUG, los receptores a la hora de consumir los mensajes buscan satisfacer 4 grupos de necesidades o deseos con las que asocian cuatro tipos de gratificación:

1. Gratificaciones cognitivas: aquellas asociadas a la necesidad de información o la pericia.

2. Gratificaciones sociales: relacionadas con las necesidades de seguridad e integración social y personal, en cuanto que refuerzan su confianza, su personalidad y amplían el grupo de amistades.

3. Gratificaciones afectivo-estéticas: relacionadas con el refuerzo de experiencias emocionales agradables y de placer.

4. Gratificaciones lúdicas: relacionadas con el deseo de entretenimiento.

A estas añadiríamos nosotros las gratificaciones políticas, vinculadas a las necesidades humanas de autorrealización personal y de equidad en el seno de comunidades que deben reconocerlos como sujetos de derechos y deberes. Pensemos en la igualdad de género y la publicidad sexista.

La nueva cultura transmediática ha contribuido a expresar «quiénes somos, reforzar nuestras relaciones personales y profesionales, fortalecer nuestras relaciones mutuas y construir comunidad y conciencia sobre los temas que nos interesan» (Jenkins et al., 2013, p. 304). Y, por ende, obligado a las marcas/organizaciones a abandonar las estrategias comunicativas top down (de arriba-abajo) y priorizar las bottom up (de abajo-arriba). Y aquí el público resulta decisivo.

Consideramos, pues, que para juzgar la transmedia advertising effectiveness resulta hoy imprescindible el enfoque de la TUG. Coincidimos aquí con Thomas E. Ruggiero, que ha realizado un estudio pormenorizado de la TUG (U\&G, en inglés) en los últimos 40 años. Ruggiero escribe: 
Theoretically and practically, for U\&G scholars, however, the basic questions remain the same. Why do people become involved in one particular type of mediated communication or another, and what gratifications do they receive from it? Although we are likely to continue using traditional tools and typologies to answer these questions, we must also be prepared to expand our current theoretical models of U\&G to include concepts such as interactivity, demassification, hypertextuality, asynchroneity, and interpersonal aspects of mediated communication. Then, if we are able to situate a "modernized" U\&G theory within this new media ecology, in an evolving psychological, sociological, and cultural context, we should be able to anticipate a highly serviceable theory for the 21st century (Ruggiero, 2000, p. 29).

Debemos identificar mediante trabajos empíricos por que y para qué la gente participa en los contenidos, usa determinados medios y se compromete con determinadas marcas. Pero, a partir de determinadas aproximaciones teóricas (Katz y otros, 1985; Urista et al., 2009, Martinez, 2011; Masullo Chen, 2011; Archer, C., y Harrigan, P., 2016), podemos catalogar las gratificaciones de los usuarios de la Web 2.0 en diez categorías: informativas, participativas, de reconocimiento, fiduiciarias, afectivas, cohesivas, empáticas, adhesivas, lúdicas y emotivas (Moya, 2017, pp. 127-129). En todo caso, proponemos tres niveles o gradientes de compromiso de los prosumidores con las marcas/organizaciones. De modo gráfico:

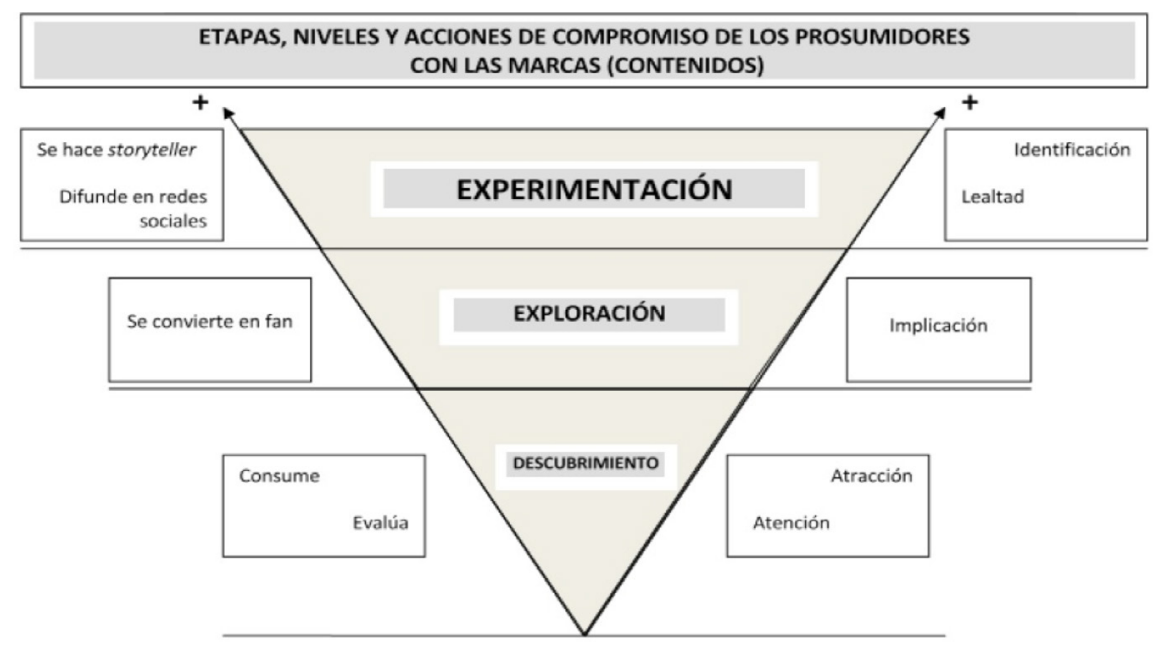

Figura 5: Niveles de compromiso y actividades del prosumidor. Elaboración propia. 
En el primer nivel agrupamos a aquellos usuarios de los cuales se obtienen datos que son aprovechados por los productores con el fin de seleccionar y personalizar el consumo de sus mensajes; y es que incluso los descubridores o merodeadores invierten en la marca, a pesar de hacerlo muchas veces de forma indirecta.

En el segundo nivel se expande la participación hacia otras etapas de construcción de mensajes/narrativas, manteniendo la guía establecida por las organizaciones y los medios, limitando, por ende, el rango de posibilidades narrativas que se ofrece a los usuarios para su consumo y/o co-creación. El usuario deviene agente activo de la organización, por lo que su participación es más elevada. Explora.

Finalmente en la tercera gradiente, aumenta el engagement y la inversión emocional, axiológica e intelectual pasa a ser avanzada. Realmente hay una identificación con la marca/organización. Crean o modifican los mensajes, las historias, su distribución y circulación. El contenido pasa a formar parte de una conversación que evoluciona conforme ellos los van difundiendo a través de los medios digitales, especialmente, las redes sociales. Hasta innovan.

En 2011, Golovinski introdujo un concepto que representa bien el tercer nivel de compromiso: common gurus, usuarios que se convierten en verdaderos atractores digitales, pues viralizan sus contenidos en Internet. Puede hablarse, incluso, de que ellos van un paso más allá del prosumo. Prodiseñan (Hernández Serrano, 2017, pp. 81-82). En este sentido, podemos afirmar con Eric von Hippel Hippel, (2005), que las redes sociales, desprofesionalizadas -redes paritarias- son redes de innovación construidas horizontalmente (bottom up):

Les réseaux d'innovation par les utilisateurs peuvent fonctionner de manière complètement autonome par rapport aux entreprises quand les conditions suivantes sont réunies : 1 ) un noyau d'utilisateurs a une forte incitation individuelle pour innover ; 2) une partie des utilisateurs sont prêts à partager, révéler leur innovation ; 3) la diffusion de l'innovation portée par les utilisateurs est peu coûteuse (et concurrence alors les offres payantes). En innovant dans des réseaux, les utilisateurs fabriquent des innovations parfaitement adaptées à leurs besoins, ce qui accroît leur satisfaction. (Beaudouin, 2011, p. 133). 
Gráficamente, tendríamos la gradiente:

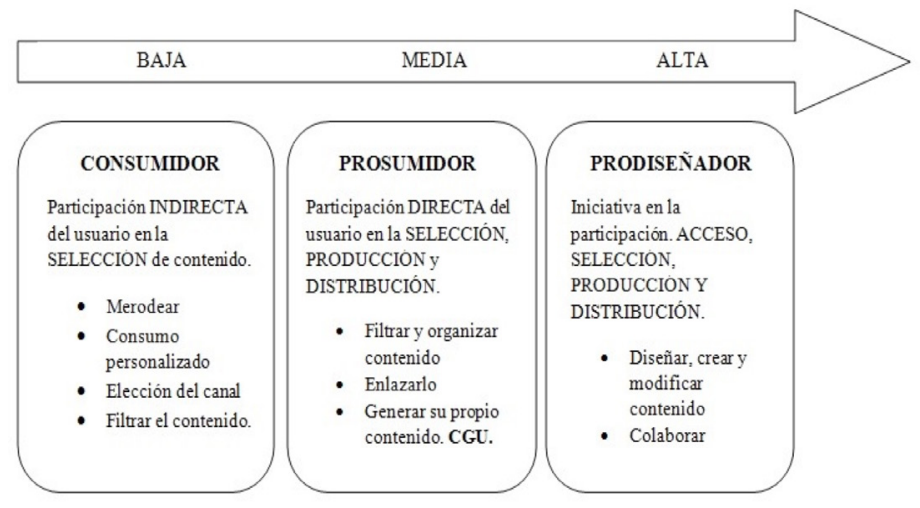

Figura 6: Gradiente de compromiso y participación en la marca. Elaboración propia.

El perfil de máxima implicación debe constituir hoy el epicentro, la top priority, de los esfuerzos comunicativos y publicitarios, pues una vez que son identificados, visibilizados, los nuevos stakeholders permiten definir, y ajustar finamente, los mensajes (el contenido), y como consecuencia convertir en eficaz las estrategias organizativas. Máxime cuando conocemos la «ley de vecindad» que exhiben todas las redes libres de escala.

En efecto, Damon Centola en su trabajo «The spread of Behavior in an Online Social Network Experiment» (2010), ha demostrado, tras estudiar la conducta de una comunidad de usuarios de Internet y las redes sociales interesados en temas de salud (con la particularidad de que cada usuario puede ver la actividad y opiniones de unos pocos miembros de la red, que son elegidos directamente por el autor del trabajo, que el clustering (agrupación) resulta fundamental: los vecinos de un determinado actor hiperenlazado son también vecinos entre sí. Centola concluye, por tanto, que es mucho más efectivo transmitir una idea o un cambio de comportamiento en redes con alto clustering ${ }^{7}$. Por así decir, por contagio. Evidentemente, este efecto $\mathrm{Mateo}^{8}$ tiene especial relevancia en la eficacia publicitaria, porque el análisis de la distribución asimétrica de oportunidades para hacer circular información y convertirse en atractor digital pasa a ser el objetivo estratégico fundamental de las organizaciones. Su cumplimiento es la única garantía hoy de las dinámicas comunicativas expansivas de las empresas o instituciones. 


\section{Conclusiones}

La revolución tecnocomunicativa digital es hoy ya un hecho. Ha propiciado entornos participativos y colaborativos y hecho emerger un nuevo actor social: el pronet@rio, cuyo correlato económico es el prosumidor. En ambos casos, estamos ante ciudadanos comunes que, gracias a los nuevos medios digitales de comunicación, han obligado a las organizaciones y/o marcas a conocer sus opiniones, intereses y valores.

En todo caso, las organizaciones han encontrado también en esos entornos nuevas oportunidades de negocio. Con las nuevas herramientas han sido capaces de entablar conversaciones con los nuevos actores hasta hacerlos copartícipes de sus estrategias comunicativas. Los prosumidores han devenido, así, auténticos stakeholders. Sus aportaciones, muchas veces intangibles, llegan a ser tan importantes para las marcas/ empresas como las económicas que realizan los accionistas.

Hemos mantenido, desde un enfoque sistémico de la comunicación que la narrativa transmedia presenta en este nuevo contexto comunicativo una ventaja que las organizaciones han comenzado a aprovechar. Sin embargo, el éxito conversacional con los prosumers pasa por conocer sus motivaciones y gratificar adecuadamente la inversión emocional o intelectual que ellos realizan. Hemos presentado, en este sentido, un modelo conceptual, que enraíza con los presupuestos de una teoría de los usos y gratificaciones, planteada a finales de los setenta del siglo pasado y que posibilita una gradación del compromiso (engagement) con la marcas, que va desde la simple atracción a la plena identificación, verdadero objetivo publicitario, porque termina convirtiendo a las mismas redes mediáticas por las que circulan las historias en verdaderas redes de innovación y diseño de marca.

\section{Referencias}

Archer, C., y Harrigan, P. (2016). Prosumers with passion: Learning what motivates bloggers as digital influencer stakeholders. PRism 13 (1). http://www. prismjournal.org/homepage.html 
Baro, M. (2011). Jerarquización de stakeholders para la construcción del capital social de las organizaciones. Mediaciones Sociales, 9 (II), 135-162. D0I: 10.5209/rev_MES0.2011.n9.3801.

Beaudouin, V. (2011). Prosumer. Communications, Cultures du numérique, 88, 131139.

Berdahl, A., Torney, C., Loannou, C., Faira, J. y Couzin, L. (2013). Emergent Sensing of Complex Environments by Mobile Animal Groups. Science, 339 (6119), 574576.

Bernays, E. (2008). Propaganda. Barcelona: Melusina.

Boccaletti, S. et al. (2006). Complex Networks: Structure and Dynamics. Physic Reports, 424, 175-308.

Castells, M. (2015). Networks of Outrage and Hope: Social Movements in the Internet Age. Malden: Polity Press.

Centola, D. (2010). The spread of Behavior in an Online Social Network Experiment. Science, 329 (5996), 1194-1197.

Christakis, N. y Fowler, J. (2010). Social Network Sensors for Early Detection of Contagious Outbreaks. PLOS ONE, 5(9), 1-8. doi:10.1371/journal.po-ne.0012948. Dowd, T. (2015). Transmedia: One Story-Many Media. Florida: CRC Press.

Dutton, W.H. (2009) The Fifth Estate Emerging through the Network of Networks. Prometheus: Critical Studies in Innovation, 27 (1), 1-15.

Fernández, J. y Bajo, A. (2012). La Teoría del Stakeholder o de los Grupos de Interés, pieza clave de la RSE, del éxito empresarial y de la sostenibilidad. aDResearch ESIC, 6 (2), 130-143.

Freeman, E. (1984). Strategic management: A stakeholder approach. Boston: Pitman.

- (1994) The Politics of Stakeholder Theory: Some Future Directions. Business Ethics Quarterly, 4 (4), 409-421.

- (2003). What Stakeholder Theory Is Not. Business Ethics Quarterly, 13 (4), 479502.

Friedman, M. (1962). Capitalism and Freedom. Chicago: The Universite of Chicago Press. Gibert, J. y Correa, B. (2001). La teoría de la autopoiesis y su aplicación en las ciencias sociales. Cinta Moebio, 12, 175-193.

Golovinski, M. (2011). Event 3.0: How Generation Y and Z Are Re-Shaping the Events Industry. Carolina: Lulu.com 
Groh S. et al. (2015). Swarm Electrification: Investigating a Paradigm Shift Through the Building of Microgrids Bottom-up. En: Groh, S. (ed.), Decentralized Solutions for Developing Economies. Berlín: Springer.

Han, B. (2014). Psicopolítica. Neoliberalismo y nuevas técnicas de poder. Barcelona: Herder.

Hernández , M.J., Renés, P., Graham, G. y Greenhill, A. (2017). Del prosumidor al prodiseñador: el consumo participativo de noticias. Comunicar, 50 (XXV), 77-87.

Herrero, P., Ramos, M., y Nó, J. (2016). Los menores como usuarios creadores en la era digital: del prosumer al creador colaborativo. Revisión teórica 19722016. Revista Latina de Comunicación Social, 71, 1301-1322. http://www. revistalatinacs.org/071/paper/1147/67es.html

Hester, P. (2015) Analyzing Stakeholders Using Fuzzy Cognitive Mapping. Procedia Computer Science, 61, 92-97.

Hippel. E. (2005). Democratizing Innovation. Camdbridge, Mass: MIT Press.

Holland, J. (2004). El orden oculto: de cómo la adaptación crea complejidad. México: Fondo de Cultura Económica.

Jenkins, H. (2008). Convergence Culture. Barcelona: Paidós.

- (2010). Transmedia Storytelling and Entertainment: An Annotated Syllabus. Continuum. Journal of Media \& Cultural Studies, 24 (6), 943-958.

Jenkins, H., Ford, S., y Green, J. (2013). Spreadable media: Creating value and meaning in a networked culture. New York: New York University Press. http:// journal.transformativeworks. org/index.php/twc/article/view/525/410

Jenkins, H., Mizuko, I. y Danah B. (2016). Participatory Culture in a Networked Era. A Conversation on Youth, Learning, Commerce and Politics, Cambridge: Polity Press.

Jenkins et al, (2016), Participatory Culture in a Networked Era: A Conversation on Youth, Learning, Commerce, and Politics. Nueva Jersey: Wiley

Jiang, Ch. Et al. 2017: Information Credibility Modeling in Cooperative Networks: Equilibrium and Mechanism Design. Selected Areas in Communications IEEE Journal on, vol. 35, pp. 432-448.

Katz, E, Blumler, J. y Gurevitch, M. (1973). Uses and Gratifications Research. The Public Opinion Quarterly, 37 (4), 509-523.

- (1985). Usos y gratificaciones de la comunicación de masas. En: Sociología de la comunicación de masas. Ed. Moragas, M. Barcelona: Gustavo Gili. 
Kinder, M. (1991). Playing with Power in Movies, Television, and Video Games: From Muppet Babies to Teenage Mutant Ninja Turtles. Berkeley: University of California Press.

Kumar, A. y Kumar Dash, M. (2015). Fuzzy Optimization and Multi-Criteria Decision Making in Digital Marketing. Hershey: IGI Global.

Krzysztof M. (2015). Transmedial World-Building in Fictional Narratives. Image, Media Convergence and Transmedial Worlds, 22 (III), 83-96.

Martínez, F. (2011). La teoría de los usos y gratificaciones aplicada a las redes sociales. Nuevos medios, nueva comunicación. Actas II Congreso Internacional "Comunicación 3.0". Universidad de Salamanca, 461-474.

Liu,B. et al., 2012: Optimizing Information Credibility in Social Swarming Applications. IEEE Transactions on Parallel and Distributed Systems, 23/6, pp. 1147-1158.

Masullo Chen, G. (2011). Tweet this: A uses and gratifications perspective on how active Twitter use gratifies a need to connect with others. Computers in Human Behavior, 27, 755-762.

Maturana, H. y Varela, F. (2004). De máquinas y seres vivos. Autopoiesis: La organización de lo vivo. Santiago de Chile: Lumen.

Merton, R. (1968). The Matthew Effect in Science. Science, new series, 159 (3810), 56-63. https://www.unc.edu/ fbaum/teahing/articles/Merton_Science_1968.pdf Moya, E. (2012). La emergencia del pronet@riado. Revista de Filosofía, 37, 7-30. Moya, E. (2015). Redes, mentes extendidas y verdad. En: La ciudad digital. Esperanzas, riesgos y desilusiones en las redes. Ed. Fernández, V. Toledo: Ediciones de UCLM.

Moya, J. (2017). Narrativa transmedia y eficacia publicitaria. Murcia: Fundación Educa.

Rickerson, W., et al. (2014). Residential prosumers-drivers and policy options (reprosumers), IEA-RETD

Ruggiero, E. (2000). Uses and Gratifications Theory in the 21st Century. Mass communication \& society, 3 (1), 3-37.

Scolari, C. (2010). Narrativa transmediática, estrategias cross-media e hipertelevisión. En Maguregui, Piscitelli y Scolari (eds.), Lostología. Estrategias para entrar y salir de la isla. Buenos Aires: Cinema. 
Tapscott, D., y Williams, A. (2009). Wikinomics: La nueva economía de las multitudes inteligentes. Barcelona: Paidós.

Toffler, A. y Toffler, H. (2006). La revolución de la riqueza. Barcelona: Debate.

Trippi, J. (2004). The Revolution Will Not Be Televised: Democracy, the Internet, and the Overthrowof Everything. Nueva York: HarperCollins.

Urista, M., Dong, Q., y Day, D. (2009). Explaining Why Young Adults Use MySpace and Facebook Through Uses and Gratifications Theory. Human Communication. A Publication of the Pacific and Asian Communication Association, 12(2), 215-229.

Watts, D and Dodds, P. S. (2007). Influentials, networks, and public opinion formation. Journal of Consumer Research, 34, 441-458.

\section{Notas}

[1] Nutricionistas y organizaciones no gubernamentales de comercio justo han puesto en el punto de mira el aceite de palma, el aceite vegetal más consumido del mundo, pero que genera serias dudas sobre su carácter nocivo para la salud e impacto en el medio ambiente.

[2] Véase: Moya, 2012.

[3] La idea de prosumer sirvió para designar a individuos o colectivos que PROducen y conSUMEN su propio output (Toffler y Toffler, 2006, p. 221).

[4] Maturana y Varela distinguen sistemas autopoyéticos de primer, segundo y tercer orden. Los primeros se dan a nivel molecular; los segundo a nivel orgánico; los terceros a nivel sociocultural. Este sería el caso de la comunicación. (Maturana y Varela, 2004, pp. 15; 18-19; 51-52). Una discusión sobre la aplicabilidad del concepto de autopoyesis a los sistemas sociales y comunicativos, puede leerse en: Gibert y Correa (2001).

[5] Freeman, 1984; 1994 y 2003.

[6] Actualmente se desarrollan modelos matemáticos para analizar los stakeholders. El problema radica en que la distinta procedencia y variedad de las partes hace difícil un enfoque mereológico. Se necesitan enfoques sistémicos y dinámicos (Hester, 2015).

[7] Este efecto se da en las redes Barabási-Albert, esto es, libres de escala, que evolucionan siguiendo la ley de potencias. (Boccaletti, 2006, pp. 175-308).

[8] Como sabemos, el efecto Mateo es un fenómeno de acumulación progresiva de bienes o fama, del que habló por primera vez Merton (1968) y que tiene su origen en la cita del Evangelio (San Mateo, 25: 29), cuando se habla de la fe y se dice: «Porque a todo el que tiene, más se le dará». 